\title{
Numerical Modeling of Vehicles Convoy Aerodynamics
}

\author{
Lilia Kataeva ${ }^{\mathrm{a}}$, Natalia Loschilova ${ }^{\mathrm{b}}$, Dmitry Maslennikov ${ }^{\mathrm{c}}$, Anton Tumasov ${ }^{\mathrm{d}}$ \\ Nizhny Novgorod State Technical University n.a. R.E. Alekseev (NNSTU), Nizhny Novgorod, Russian Federation \\ akataeval2010@mail.ru, ${ }^{b}$ loschilovana@gmail.com, ${ }^{\mathrm{c}}$ dmitrymaslennikov@rambler.ru, ${ }^{\mathrm{d}}$ anton.tumasov@gmail.com
}

\begin{abstract}
This paper devoted to the development of the coordinate system discontinuous transformation for the simulation of multiple groups periodic motion and their aerodynamic interaction. The proposed approach reduced to the creation a separate local coordinate system for each group of bodies that is stationary relative to them. Each of the coordinate systems has effect on the corresponding subdomain. For obtaining solutions at the interface between subdomains we use linking algorithm based on the calculation of the interface points in local coordinate systems. Result of the aerodynamic interactions of periodic moving group of bodies shown and error arising from the use of the proposed approach estimated.
\end{abstract}

Keywords-numerical simulation; aerodynamics of the car; a column of vehicles

\section{INTRODUCTION}

The study of aerodynamics of single vehicle has always been important. However, after some time more attention has been paid to the more complicated scenarios of the movement. Thus, for practical purpose vehicle simulation of bodies group movement is already more than a matter of fuel efficiency and effective capacity utilization of the car, but the predictability of movement and as a consequence, traffic safety factor.

However, the modeling of cars group aerodynamics actual for racing cars too. In paper [1] considered the influence of air resistance depending on the relative position of several cars. The authors discovered that during the motion cars group one after another, cars are able to reach speed greater than the maximum speed of single vehicle, which is $320 \mathrm{~km} / \mathrm{h}$. This effect is achieved due to the formation region of rarefied air around the first car conducive to the movement of vehicle moving behind. The same effect is taking place when driving in a convoy. When driving side by side, the speed of both cars decreases significantly due to partial blocking flow, forcing the air deviates strongly, flowing around both vehicles same time.

The paper [2] is devoted to experimental research of the aerodynamic interaction of passenger cars. The paper considers the movement of the column and overtaking vehicles, the behavior of the car in the tunnel including the presence of a trailer on a rigid coupling. The author focuses on the study of the Reynolds number influence on the aerodynamic characteristics. The method of flow visualization used to identify the main effects.

Air flow directed, generally parallel to the direction of travel, is very complicates vehicle movement. In case of high speed vehicles are greatly affected by the side velocity component of air flow. Side forces are usually large enough while overtaking and may affect the control of the vehicle. These issues are discussed in [3]. The paper also demonstrates a two-dimensional simulation of the air flow collision for overtaking vehicles. The calculation results are in good agreement with the experimental data.

However, modeling the movement of several vehicles causes a number of problems related with the coordinate system. If several vehicles are moving with different velocities, any coordinate system with all the points moving with constant velocity can't provide vehicles immobility relatively to it.

For introducing a coordinate system in which coordinates of different points can have a different velocity relative to stationary observer, transformation consisting deformations or discontinuities must be used. In modern software products for modeling the relative motion of bodies group using various approaches, such as Overset Mesh [4], based on the creation of the grid associated with the moving body and interpolation between borders of this grid and background mesh. This method requires approximately equal cell sizes of overset and background meshes, which somewhat reduces the possibility of optimizing the mesh through its local refinement. In ANSYS Fluent to solve such problems are used Dynamic and Moving Mesh. This approach allows us to reconstruct the mesh in accordance with the movement of simulated objects. This way of modeling is more resource intensive, but eliminates the problem of interpolation error and stability of at the intersection of overlapping meshes [5].

In contrast to the approaches described above, in this paper a method based on discontinuous coordinate transformation proposed, allowing solving the problem of aerodynamics of periodically moving bodies group.

\section{THE OBJECT OF RESEARCH}

Movement of vehicles on multi-lane road on the assumption that the column of identical cars can move along each lane considered. Fig. 1 shows a diagram of two-lane traffic. Every lane corresponds to its computational subdomain; however exact match between them is not required. Every subdomain may include only vehicles moving with same direction and velocity.

Repeating periodically region $\Gamma i$ on each of the $n$ subdomains considered. The length of each region $\Gamma i$ is equal to $\mathrm{L}$, which corresponds to the sum of the length and the distance between the bodies moving in a group within the subdomain. In order to preserve the periodicity proceeding processes, it is necessary that the sum of the distance 
between the vehicles and their length is always constant at the all subdomains.

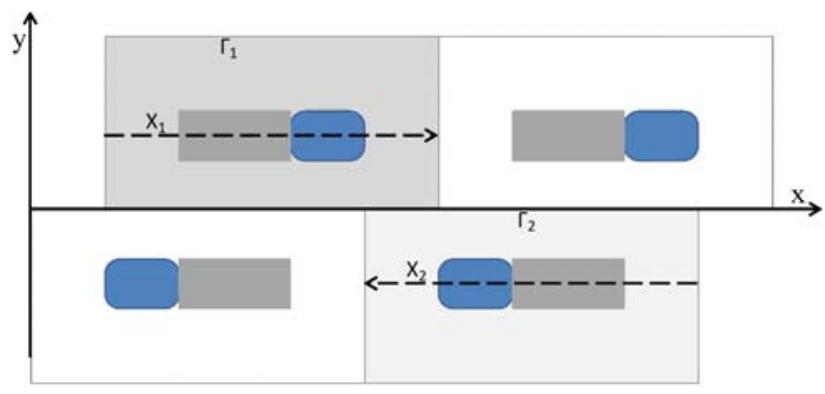

Figure 1. Diagram of multi-lane traffic

To solve the problem using converting the $\mathrm{x}$ coordinate, several coordinate systems introduced, and the y coordinate is not converted. On each subdomain a local coordinate system moving with its body introduced. For i-th subdomain coordinate transformation has the form

$$
x_{i}=x-U_{i} t
$$

Where $\mathrm{i}$-th subdomain defined by its coordinates $y_{i-1} \leq y \leq y_{i}, U_{i}$ - velocity of bodies group moving at i-th subdomain. Strights $y=y_{i}$, where $i=1,2, \ldots, n$ - are either boundaries of computational domain or common boundaries of adjoining subdomains. Suppose system coordinate $\tilde{x} O y$ have abscissa defined by

$$
\tilde{x}=\left\{\begin{array}{l}
x_{1}, \text { npu } y_{0}<y<y_{1} \\
x_{2}, \text { npu } y_{1}<y<y_{2} \\
\cdots \\
x_{n}, \text { npu } y_{n-1}<y<y_{n}
\end{array}\right.
$$

So, the coordinate system $\tilde{x} O y$ linked to every of subdomains, and all bodies are stationary relative to it, however transformation (1) is discontinuous at boundaries $y=y_{i}(i=0,1, \ldots, n)$. However, these discontinuities are removable, since they do not have physically based sources of jumps solution. Algorithm for finding the numerical solution around such discontinuities should be given particular attention to. Due to the movement of local coordinate systems, absolute value of point B abscissa can exceed L, so periodicity of the solution has to be used.

Let point $\mathrm{A}$ is on $\mathrm{i}$-th subdomain and have coordinates $\left(a, y_{i-1}+\frac{\Delta y}{2}\right)$ in local coordinate system $x_{i} O y ; \Delta y$ - step in the coordinate $\mathrm{y}$. Assume, computation algorithm needs value in point $\mathrm{B}$ having coordinate $\left(a, y_{i-1}-\frac{\Delta y}{2}\right)$ in $x_{i} O y$ in order to use numeric scheme at point $\mathrm{A}$. Corresponding to coordinate transformation formula (1), point B have coordinates $\left(a+U_{i} t, y_{i-1}-\Delta y\right)$ in system $x_{i} O y$ and coordinates $\left(a+\left(U_{i}-U_{i-1}\right) t, y_{i-1}-\Delta y\right)$ in $x_{i-1} O y$.

Point B corresponds to the point B0, belonging subdomain $\quad$ i-1, and has coordinates $\left(\bmod \left(a+\left(U_{i}-U_{i-1}\right) t, L\right), y_{i-1}-\Delta y\right)$ in local coordinate system $x_{i-1} O y$, where $\bmod (x, y)=x-y\left\lfloor\frac{x}{y}\right\rfloor$. Point B0 belongs to (i-1)-th subdomain, but due to the fact that the relative shift of $i$-th and (i+1)-th subdomain is not a multiple step-coordinate, interpolation by coordinate $\mathrm{x}$ is required. In this paper, linear interpolation was used for calculations.

In this paper, the approach described above was used to simulate aerodynamics of the group of moving bodies. For the aerodynamics simulation RANS (Reynolds-averaged Navier-Stokes) equations with k- $\omega$ turbulence model used.

In view of the fact that all these processes - isothermal equation of energy conservation is not considered. Thus, the Navier-Stokes equations in a Cartesian coordinate system in the integral form for a given volume $\mathrm{V}$ with the surface of a for solutions of these problems can be represented as

$$
\begin{array}{r}
\frac{\partial}{\partial t}\left(\int_{\widetilde{V}} W_{\chi} d \widetilde{V}+\oint[F-G] \cdot d \widetilde{a}\right)=\int_{\widetilde{V}} H d \widetilde{V} \\
W=\left[\begin{array}{l}
\rho \\
\rho v
\end{array}\right], F=\left[\begin{array}{l}
\rho v \\
\rho v \otimes v+p I
\end{array}\right], G=\left[\begin{array}{l}
0 \\
T
\end{array}\right], H=\left[\begin{array}{l}
0 \\
f_{g}
\end{array}\right], \\
\text { Where } \rho-\text { gas density, } \mathrm{v}-\text { gas phase velocity } \\
p=\frac{\rho R T_{e}}{M}-\text { pressure, } d \widetilde{V}=\alpha_{i} d V \text {, where } \alpha_{i} \text { - volume }
\end{array}
$$
fraction of phase $\mathrm{i}, \mathrm{T}-$ viscous stress tensor, fg - force of gravity, I - identity matrix, $\mathrm{R}=8.3144621 \mathrm{~m} 2 \mathrm{~kg} \mathrm{~s}-2 \mathrm{~K}-1$ mol-1 - universal gas constant; $\mathrm{M}=28.98 \mathrm{~kg} / \mathrm{mol}$ - molar mass of air; $\mathrm{Te}=300 \mathrm{~K}-$ ambient temperature. In order determine the viscous stress tensor Boussinesq approximation is used

$$
T=\mu\left(\nabla v+\nabla v^{T}-\frac{2}{3}(\nabla \cdot v) I\right)
$$

Where ${ }^{\mu}$ - dynamic viscosity.

\section{RESULTS}

Modeling was carried out in two-dimensional formulation. To test the efficiency of the numerical scheme and algorithm that implements the link between the subdomains as described earlier, the simulation of flowing around of bodies group at a speed of $30 \mathrm{~m} / \mathrm{s}$ along the axis $\mathrm{X}$ was carried out. Flowing around body had the form of a rectangle with sides 3 and 2 meters. It is assumed that adjacent subdomain have no bodies on it, however in order to test the boundaries linking algorithm, it is assumed that this subdomain moves at a velocity of $30 \mathrm{~m} / \mathrm{s}$ in the opposite direction. As a result, the beginning of the body movement, 
there is a change of pressure and elastic waves formed. Currently, as it can be seen the beginning of formation air flows in front of and behind the body. Elastic wave does not change its shape, despite the presence of subdomain boundaries, as demonstrated in Fig. 2. Fig. 2 (b) shows that the elastic wave passes through lateral boundaries without the observed error. Elastic wave with reduced pressure circulates through the left periodic boundary on the right one.

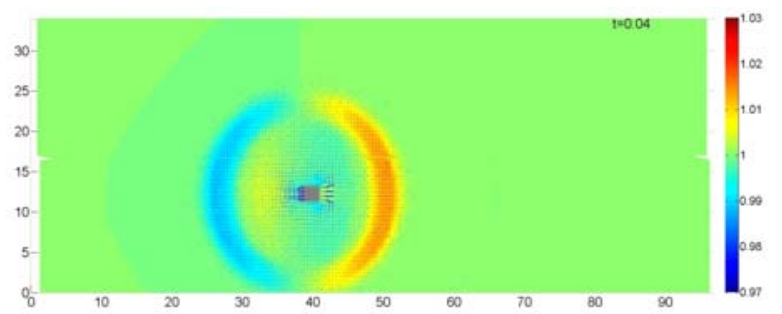

a)

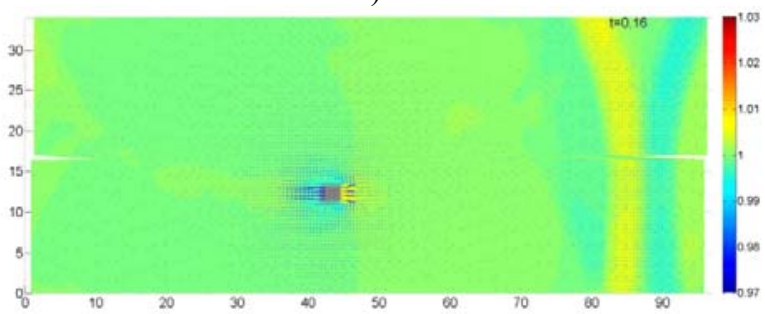

b)

Figure 2. Aerodynamics of periodically moving group: a) $\mathrm{t}=0,04 \mathrm{sec}, \mathrm{b}) \mathrm{t}=0,16 \mathrm{sec}$.

As body moves the length of flow behind it increases, which leads to the formation of Karman vortex street it clearly demonstrated in Fig. 3. Despite the large distance among neighboring bodies in a group, the flows formed by them, reach behind the moving body. Fig. 3 (a) shows the result of simulation of aerodynamics body in Matlab based on the algorithm described above in this paper. Fig. 3 (b) shows the result of a similar numerical calculation in the environment Star CCM + considering k- turbulence model. This calculation used mesh of the same structure and size as in the calculation using Matlab. As seen from the results in Fig. 3, the aerodynamic flow pattern is formed at the wrap around an object, has a similar structure and characteristic sizes. The results obtained in Matlab and Star CCM + have similar aerodynamic flow pattern for the body of rectangular shape considering same initial conditions and parameters of the computational grid, as illustrated on the Fig. 3.

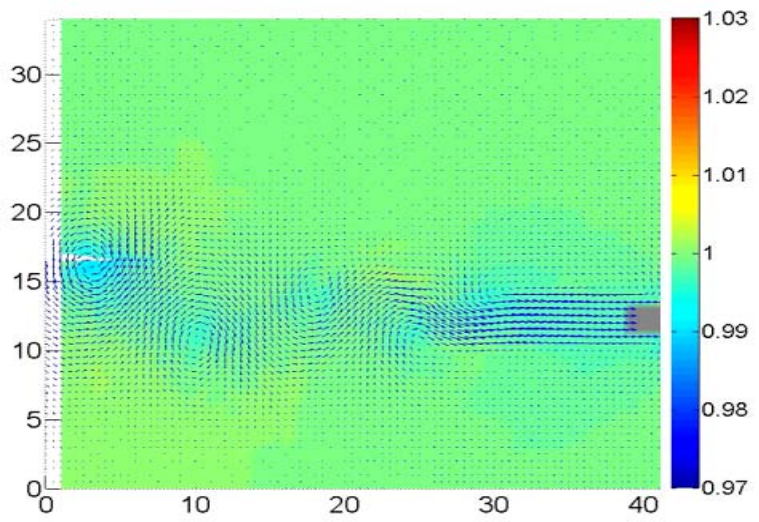

a)

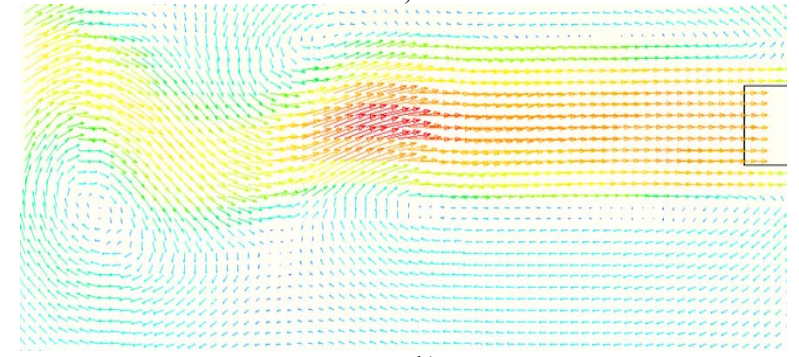

b)

Figure 3. Aerodynamics of periodically moving group at time 3,3 seconds. a - result of simulation of aerodynamics body in Matlab based on the algorithm described in this paper, $b$ - result of a similar numerical calculation in the environment Star CCM +

Even under small perturbations can cause change in the solution in aerodynamic problems, but the flow structure and scale of processes do not change significantly. Therefore, to estimate the error force acting on the body was analyzed. Positive values Fx corresponds to the direction of body movement, Fy directed to the left relative to it. The magnitude and direction of the force depends on the time significantly, undergoing considerable fluctuations. In this regard, the standard error estimation methods would overestimate error, so statistical approach was used.

At the first stage of the analysis calculation of the values Fx and Fy each time step in the solution of the flow problem was carried out. Further, it was assumed that Fx and Fy at random time with equal probability can take the value measured at any time step. On the basis that distribution function $\Phi\left(F_{x}\right)$ и $\Phi\left(F_{y}\right)$ of these forces were obtained.

Their graphs are shown in Fig. 4. The blue line shows the function of distribution of forces in the flow simulation without the use of coordinate transformations, the red line using the coordinate transformations. Since the problem is two-dimensional, it is assumed that the process does not depend on the vertical coordinates. In this connection, $\mathrm{Fx}$ and Fy have dimension $\mathrm{N} / \mathrm{m}$.

As seen from Fig. 4 results, the results obtained using the coordinate transformation, and without agree sufficiently well. 


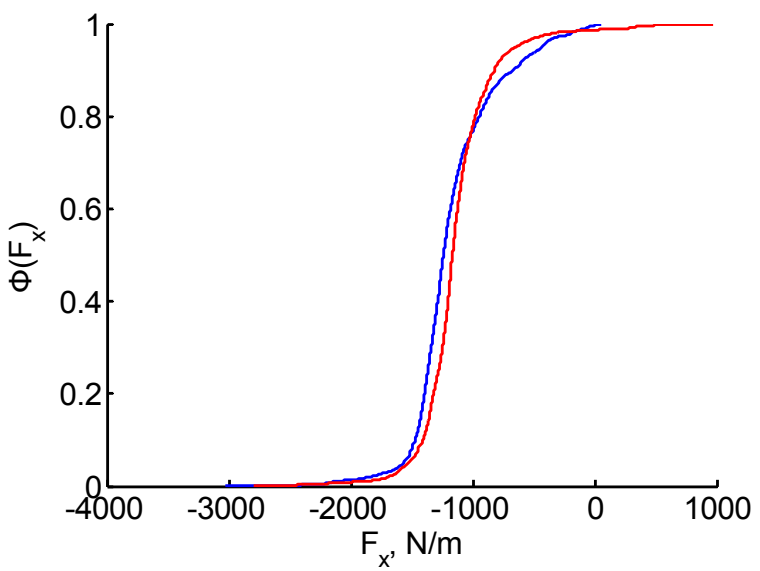

a)

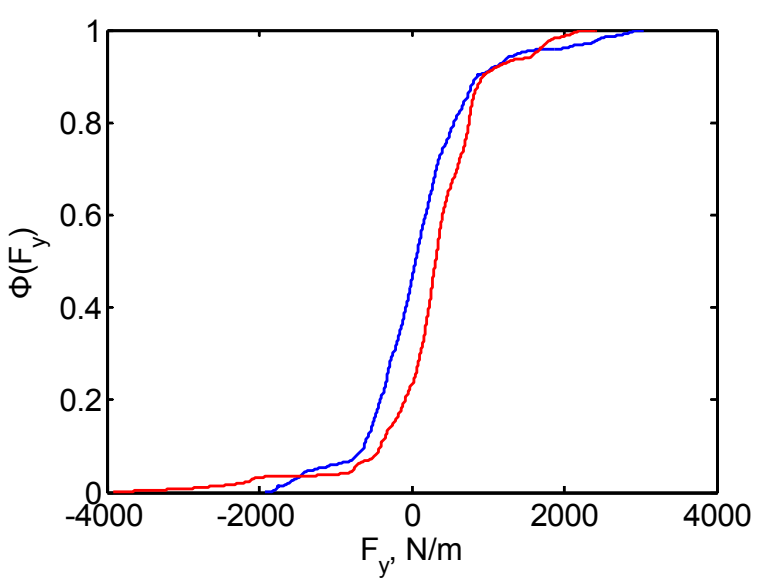

b)

Figure 4. The distribution functions of the forces acting on the rigid body: $\mathrm{a}$ - in the direction of motion, $\mathrm{b}$ - lateral force

\section{SUMMARY}

As noted above, for the solution of aerodynamics of one bodies group did not need the use the discontinuous coordinate transformation. Nevertheless, according to simulations carried out, discontinuous transformation has no significant impact on forces acting on flowing around body.

It should be noted that suggested in this paper approach can be used for CFD-system calculations due to the settings interface between the boundaries of the subregions, which can lead to less errors due to more efficient methods of interpolation. Main limitation of suggested algorithm is impossibility of modeling the body motion with variable speed.

\section{ACKNOWLEDGMENT}

The research was done with the financial support of Ministry of Education and Science of the Russian Federation in the frame of the complex project "The establishment of the advanced technology production of ecological and resources-economy LCV" under the contract №02.G25.31.006 from 12.02.2013 (Governmental Regulation №218 from 09.04.2010).

\section{REFERENCES}

[1] Information on http:/www.jcs-group.com/racin/guide/draft.html

[2] F. Ahmed, Abdel Azim, and Ahmed F. Abdel Gawad A Flow Visualization Study of the Aerodynamic Interference between Passenger Cars / SAE 2000 World Congress, Detroit, (2000).

[3] Tank Nilesh R1 and R. Thundil Karuppa Raj Numerical Simulation of Aerodynamic forces acting on Passenger Vehicle While Overtaking / // Reserch Journal of Recent Sciences. 2012. Vol. 1(12), p. 52-63 (2012).

[4] Information on

http://www.cd-adapco.com/products/star-ccm $\% \mathrm{C} 2 \% \mathrm{AE} /$ overset

[5] Information on

http:/www.ansys.com/Products/Simulation+Technology/Fluid+Dyna mics/

[6] Fluid+Dynamics+Products/ANSYS+Fluent/Features 